OCU-PHYS 249

AP-GR 35

\title{
Black Holes on Eguchi-Hanson Space in Five-Dimensional Einstein-Maxwell Theory
}

\author{
Hideki Ishihara*, Masashi Kimura ${ }^{\dagger}$, Ken Matsuno ${ }^{\ddagger}$, and Shinya Tomizawa ${ }^{\S}$ \\ Department of Mathematics and Physics, \\ Graduate School of Science, Osaka City University, \\ 3-3-138 Sugimoto, Sumiyoshi, Osaka, 558-8585, Japan
}

(Dated: June 16, 2018)

\begin{abstract}
We construct a pair of black holes on the Eguchi-Hanson space as a solution in the fivedimensional Einstein-Maxwell theory.

PACS numbers:
\end{abstract}

\footnotetext{
* E-mail: ishihara@sci.osaka-cu.ac.jp

$\dagger$ E-mail: mkimura@sci.osaka-cu.ac.jp

‡E-mail: matsuno@sci.osaka-cu.ac.jp

$\S$ E-mail: tomizawa@sci.osaka-cu.ac.jp
} 
In this brief report, we construct a pair of black holes on the Eguchi-Hanson space as a solution in the five-dimensional Einstein-Maxwell theory. The metric and the gauge potential one-form are given by

$$
\begin{aligned}
& d s^{2}=-H^{-2}(r, \tilde{\theta}) d T^{2}+H(r, \tilde{\theta}) d s_{\mathrm{EH}}^{2} \\
& \boldsymbol{A}= \pm \frac{\sqrt{3}}{2} H^{-1}(r, \tilde{\theta}) d T
\end{aligned}
$$

with

$$
\begin{aligned}
& d s_{\mathrm{EH}}^{2}=\left(1-\frac{a^{4}}{r^{4}}\right)^{-1} d r^{2}+\frac{r^{2}}{4}\left[\left(1-\frac{a^{4}}{r^{4}}\right)(d \tilde{\psi}+\cos \tilde{\theta} d \tilde{\phi})^{2}+d \tilde{\theta}^{2}+\sin ^{2} \tilde{\theta} d \tilde{\phi}^{2}\right], \\
& H(r, \tilde{\theta})=1+\frac{m_{1}}{r^{2}-a^{2} \cos \tilde{\theta}}+\frac{m_{2}}{r^{2}+a^{2} \cos \tilde{\theta}},
\end{aligned}
$$

where $a$ and $m_{j}(j=1,2)$ are constants, $0 \leq \tilde{\theta} \leq \pi, 0 \leq \tilde{\phi} \leq 2 \pi / n$, ( $n$ : natural number $)$ and $0 \leq \tilde{\psi} \leq 2 \pi$.

The equation (3) is the metric form of the Eguchi-Hanson space 1]. The Eguchi-Hanson space has an $S^{2}$-bolt at $r=a$, where the Killing vector field $\partial / \partial \tilde{\psi}$ vanishes. The function $H(r, \tilde{\theta})$ is a harmonics on the Eguchi-Hanson space (3).

As is seen later, two black holes are located on the north pole $(\tilde{\theta}=0)$ and the south pole $(\tilde{\theta}=\pi)$ on the $S^{2}$-bolt. The asymptotic behaviour of the metric (1) near the spatial infinity $r \rightarrow \infty$ becomes

$$
d s^{2} \simeq-d T^{2}+d r^{2}+\frac{r^{2}}{4}\left[d \tilde{\theta}^{2}+\sin ^{2} \tilde{\theta} d \tilde{\psi}^{2}+(d \tilde{\phi}+\cos \tilde{\theta} d \tilde{\psi})^{2}\right] .
$$

Since the $T=$ const. surface has the structure of lens space $L(2 n ; 1)$, this solution is asymptotically locally flat. The Komar mass, $M_{\text {Komar }}$ and the total electric charge, $Q$ at the spatial infinity are given by

$$
M_{\text {Komar }}=\frac{\sqrt{3}}{2}|Q|=\frac{3 \pi\left(m_{1}+m_{2}\right)}{8 n G},
$$

where $G$ is the five-dimensional gravitational constant.

In order to clarify the physical properties of the solution, we introduce the coordinates as follows [2],

$$
\begin{aligned}
& R=a \sqrt{\frac{r^{4}}{a^{4}}-\sin ^{2} \tilde{\theta}}, \quad \tan \theta=\sqrt{1-\frac{a^{4}}{r^{4}}} \tan \tilde{\theta}, \quad \phi=\tilde{\psi}, \quad \psi=2 \tilde{\phi} . \\
& (0 \leq \theta \leq \pi, \quad 0 \leq \phi \leq 2 \pi, \quad 0 \leq \psi \leq 4 \pi / n)
\end{aligned}
$$

Then, the metric takes the form of

$$
d s^{2}=-H^{-2}(R, \theta) d T^{2}+H(R, \theta) d s_{\mathrm{EH}}^{2},
$$


with

$$
\begin{aligned}
& d s_{\mathrm{EH}}^{2}=V^{-1}(R, \theta)\left[d R^{2}+R^{2}\left(d \theta^{2}+\sin ^{2} \theta d \phi^{2}\right)\right]+V(R, \theta)\left(\frac{a}{8} d \psi+\omega_{\phi} d \phi\right)^{2}, \\
& H(R, \theta)=1+\frac{m_{1} / a}{\left|\boldsymbol{R}-\boldsymbol{R}_{1}\right|}+\frac{m_{2} / a}{\left|\boldsymbol{R}-\boldsymbol{R}_{2}\right|}, \\
& V^{-1}(R, \theta)=\frac{a / 8}{\left|\boldsymbol{R}-\boldsymbol{R}_{1}\right|}+\frac{a / 8}{\left|\boldsymbol{R}-\boldsymbol{R}_{2}\right|}, \\
& \omega_{\phi}(R, \theta)=\frac{a}{8}\left(\frac{R \cos \theta-a}{\sqrt{R^{2}+a^{2}-2 a R \cos \theta}}+\frac{R \cos \theta+a}{\sqrt{R^{2}+a^{2}+2 a R \cos \theta}}\right),
\end{aligned}
$$

where $\boldsymbol{R}=(x, y, z)$ is the position vector on the three-dimensional Euclid space and $\boldsymbol{R}_{1}=$ $(0,0, a), \boldsymbol{R}_{2}=(0,0,-a)$. The metric (9) is the Gibbons-Hawking two-center form of the Eguchi-Hanson space [2, 3]. It is manifest in the coordinate that the space has two nut singularities at $\boldsymbol{R}=\boldsymbol{R}_{j}$ where the Killing vector field $\partial / \partial \psi$ vanishes.

The function $H(R, \theta)$ is the harmonics given by (44) on the Eguchi-Hanson space in the Gibbons-Hawking coordinates (92). The harmonics $H(R, \theta)$ converts nut singularities on the Eguchi-Hanson space to regular hypersurfaces in the total spacetime. Since each hypersurface $\boldsymbol{R}=\boldsymbol{R}_{j}$ becomes a Killing horizon with respect to the Killing vector field $\partial / \partial T$, and each three-dimensional section of them with $T=$ const. has finite area, then the hypersurfaces $\boldsymbol{R}=\boldsymbol{R}_{j}$ are event horizons.

Since the Kretschmann invariant $R_{\mu \nu \rho \lambda} R^{\mu \nu \rho \lambda}$ has a finite value on each horizon, we see that the geometry on the horizons is regular. Even if one of $m_{j}$ (for an example $m_{2}$ ) vanishes, which corresponds to a single black hole and a naked nut charge with the value $a / 8$, the horizon is regular. The spacetime is regular in the case of $n=1$ but it has a nut singularity at $\boldsymbol{R}=\boldsymbol{R}_{2}$ in the case of $n \geq 2$.

The induced metric on the spatial cross section of the $j$-th horizon is given by

$$
d s_{\text {Horizon }}^{2}=\frac{m_{j}}{8}\left[d \theta^{2}+\sin ^{2} \theta d \phi^{2}+(d \psi+\cos \theta d \phi)^{2}\right], \quad(0 \leq \psi \leq 4 \pi / n)
$$

which is the lens space $L(n ; 1)$. The geometry near horizons of this solution is similar to the multi-black hole solutions on the Gibbons-Hawking multi-instanton space[4], but the asymptotic structures are different. Although both are asymptotically locally flat, the former is isotropic in four spatial dimensions while the latter has a compact dimension as same as Kaluza-Klein black holes discussed in Ref.[4, [5]. 


\section{Acknowledgements}

We thank K. Nakao and Y. Yasui for useful discussions. This work is supported by the Grant-in-Aid for Scientific Research No.14540275.

[1] T. Eguchi and A. J. Hanson, Phys. Lett. 74B, 249 (1978).

[2] M. K. Prasad, Phys. Lett. 83B, 310 (1979).

[3] G. W. Gibbons and S. W. Hawking, Phys. Lett. 78B, 430 (1978).

[4] H. Ishihara, M. Kimura, K. Matsuno, and S. Tomizawa, arXiv:hep-th/0605030.

[5] H. Ishihara and K. Matsuno, arXiv:hep-th/0510094. 